Supporting Information

\title{
Large-Scale Graphene on Hexagonal-BN Hall Elements: Prediction of Sensor Performance without Magnetic Field
}

Min-Kyu Joo ${ }^{1,2 \dagger}$, Joonggyu Kim ${ }^{2 \dagger}$, Ji-Hoon Park ${ }^{1}$, Van Luan Nguyen ${ }^{1}$, Ki Kang Kim ${ }^{3}$, Young Hee Lee ${ }^{1,2^{*}}$, and Dongseok $\operatorname{Suh}^{2 *}$

${ }^{1}$ Center for Integrated Nanostructure Physics (CINAP), Institute for Basic Science (IBS), Suwon 16419, Republic of Korea

${ }^{2}$ Department of Energy Science, Sungkyunkwan University, Suwon 16419, Republic of Korea.

${ }^{3}$ Department of Energy and Materials Engineering, Dongguk University, Seoul 04620, Republic of Korea

$\uparrow$ These authors contributed equally to this work.

*Corresponding authors’ emails: leeyoung@skku.edu (Y.H.L.) and energy.suh@skku.edu (D.S.) 


\section{Additional data graphs for the characterization of GHE with large-area CVD-grown graphene/h-BN heterostructure}

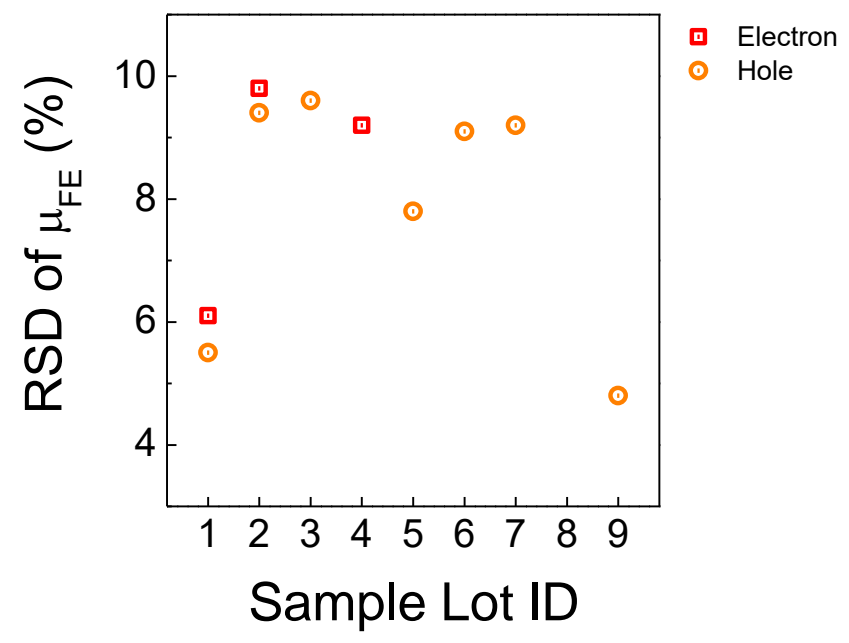

Figure S1. Relative standard deviation (RSD) of the field-effect mobility $\left(\mu_{\mathrm{FE}}\right)$ of graphene Hall element (GHE) at different sample lot ID. Several GHE devices were test at each lot to calculate the RSD of $\mu_{\mathrm{FE}}$, and the total number of GHE devices under test was 32 . The best RSD value is approximately $5 \%$, but on average the RSD is around $8-9 \%$. This variation can be ascribed to deviations of the contact resistance, the quality of the underlying $h$ - $\mathrm{BN}$, and the position of the charge neutral point.

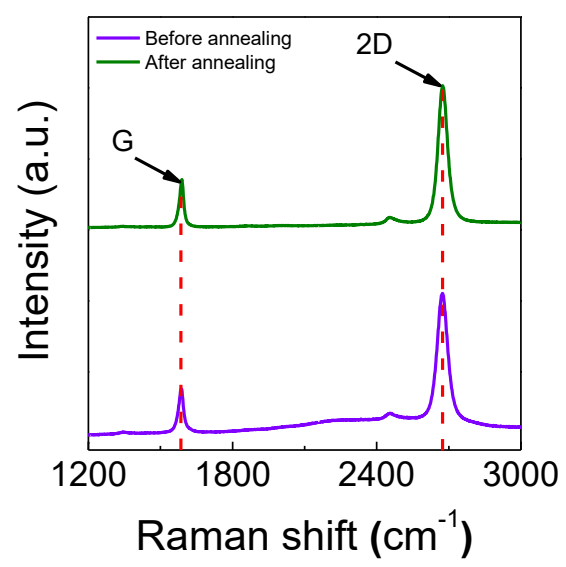

Figure S2. Post annealing effect on Raman spectroscopy of a GHE. Raman spectra of a graphene monolayer on $h$ - $\mathrm{BN} / \mathrm{SiO}_{2}$ before and after thermal annealing at $350{ }^{\circ} \mathrm{C}$ for $2 \mathrm{~h}$ under 
argon and hydrogen at flow rates of $500 \mathrm{sccm}$ and $100 \mathrm{sccm}$, respectively. The G and 2D peaks originate from the first-order Raman scattering process and the second-order two-phonon process, respectively. After thermal annealing, we did not observe blue shifts of the $\mathrm{G}$ and 2D peaks, which generally originate from the hole-doping effect in graphene. In other words, the $h$-BN thin film inserted between the graphene and oxide prevents induced doping from the substrate.

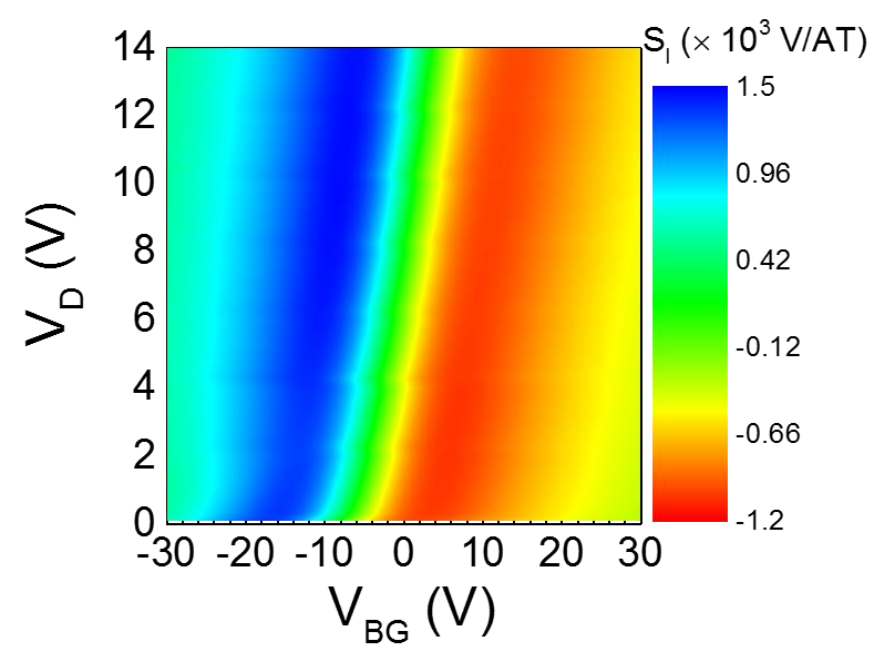

Figure S3. Calculated Hall effect current sensitivity $\left(S_{I}\right)$ of GHE. To determine the optimal electric-field bias conditions for $I_{D}$ as well as $V_{D}$ and $V_{\mathrm{BG}}$, in order to achieve the maximum value of $S_{I}\left(S_{I_{\perp} \max }\right)$ prior to Hall measurements, we propose a new analytical model that takes into account $I_{D}$ and its transconductance $\left(g_{m}=\partial I_{D} / \partial V_{\mathrm{BG}}\right.$, where $I_{D}$ and $V_{\mathrm{BG}}$ denote the drain current bias and the back gate bias, respectively) of the cross-shaped GHEs. The new model is based on the relation $S_{I}=-r_{H} \alpha g_{m} /\left(I_{D} \mathrm{Cox}\right)$, where $r_{H}, \alpha$, and $C_{\mathrm{OXX}}$ denote the Hall factor, the geometrical correction factor, and the oxide capacitance, respectively. For consistency with the main text, we choose $r_{H} \alpha=3$. Though the GHE used is different from that in main text, the trend is almost the same as in Figure 4(e), supporting our approach. This simple but powerful model leads to determining the optimal operation window for not only GHEs but also other types of Hall elements, without needing a magnetic field-based Hall effect measurement. 
(a)

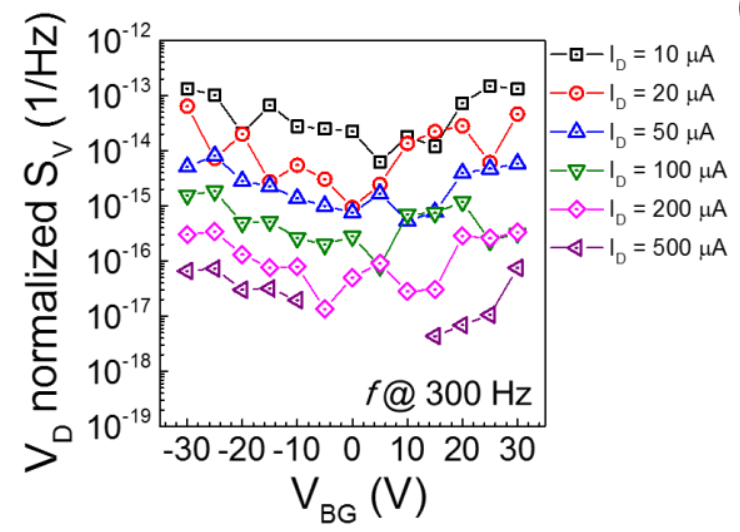

(b)

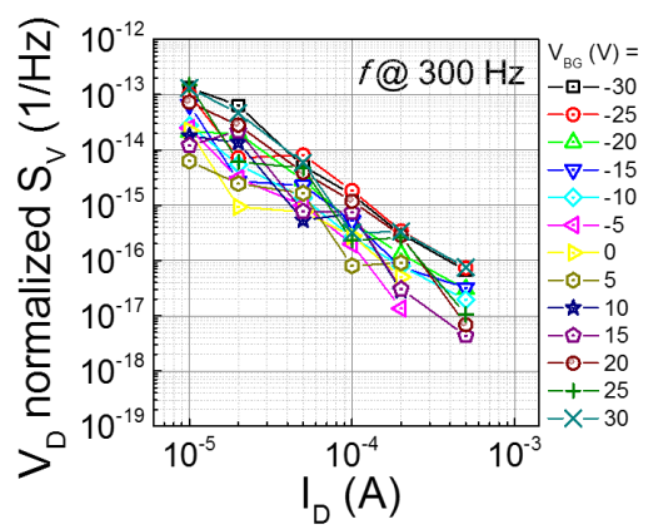

Figure S4. Voltage power spectrum $\left(S_{V}\right)$ normalized by drain voltage $\left(V_{D}\right)$. (a) The $V_{D^{-}}$ normalized $S_{V}$ as a function of $V_{\mathrm{BG}}$, with $I_{D}$ as a parameter. (b) The same data plotted as a function of $I_{D}$, with $V_{\mathrm{BG}}$ as a parameter.

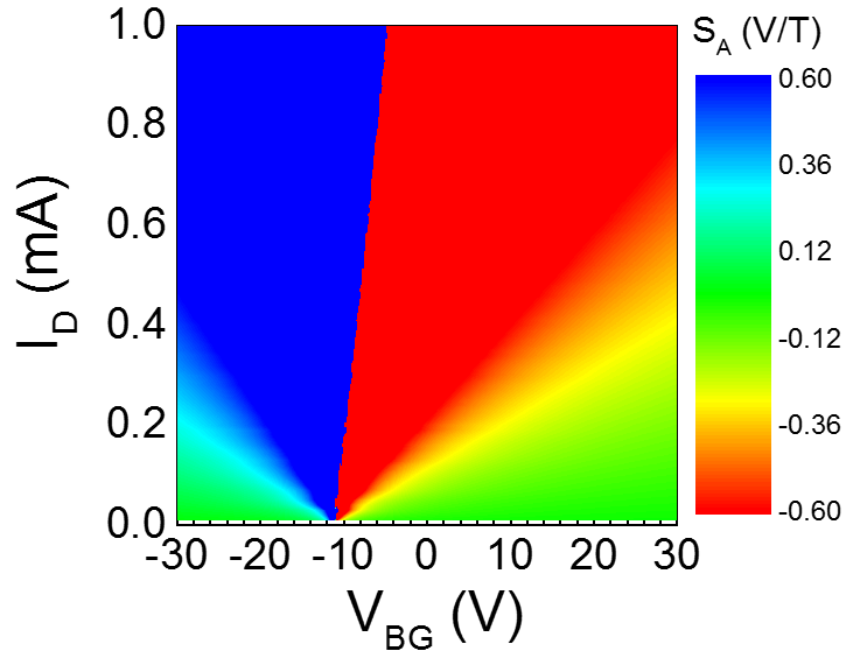

Figure S5. Numerically calculated $S_{A}$. A 2-D contour plot of absolute Hall sensitivity $S_{A}$ obtained by simply regarding transconductance $g_{m}$ as $\approx I_{D} /\left(V_{\mathrm{BG}}-V_{\mathrm{CNP}}\right)$, where $V_{\mathrm{CNP}}$ is the charge neutral point. As described in the main manuscript, the red and blue areas indicate the optimal bias boundaries for the maximum $S_{A}$ of the GHE. However, due to the approximate model of $g_{m}$ used here, there are regions of inaccuracy near $V_{\mathrm{BG}}=V_{\mathrm{CNP}}$. 


\section{B min-limitation of GHE}

On the basis of Eq. (2) in the main text (which includes the representative LF noise model), the optimal boundary conditions for the minimum magnetic resolution $B_{\min }$ can be analytically suggested. These estimates are: for (a) monolayer graphene transistors with a Hooge mobility fluctuation model (HMF), and for (b) silicon transistors with a carrier number fluctuation model $(\mathrm{CNF})$, as described in the references below ${ }^{1-3}$,

$$
\begin{gathered}
\text { HMF Case }: B_{\min }=\frac{\sqrt{S_{V}}}{S_{A}} \approx \frac{\sqrt{\frac{V_{D}^{2} e \alpha_{H}}{f L W C_{O X}\left(V_{B G}-V_{C N P}\right)}+4 k_{B} T \frac{V_{D}}{I_{D}}}}{\left(r_{H} \alpha \frac{g_{m}}{C_{O X}}\right)} \\
\text { CNF Case }: B_{\min }=\frac{\sqrt{S_{V}}}{S_{A}}=\frac{\sqrt{\frac{V_{D}^{2} e^{2} k_{B} T N_{S T}}{f W L C_{O X}^{2}}\left(\frac{g_{m}}{I_{D}}\right)^{2}+4 k_{B} T \frac{V_{D}}{I_{D}}}}{\left(r_{H} \alpha \frac{g_{m}}{C_{O X}}\right)}
\end{gathered}
$$

where $\alpha_{H}$ and $N_{\mathrm{ST}}$ are Hooge's constant and the surface trap density, respectively. Provided that $\alpha_{H}$ and $N_{\mathrm{ST}}$ are constant and thermal noise is negligible compared to flicker noise, $B_{\min }$ here will be proportional to $V_{D} /\left(I_{D} g_{m}\right)^{0.5}$ for GHE and $V_{D} / I_{D}$ for silicon-based Hall elements. As a consequence, our newly-suggested analytical model may be applicable to other types of Hall elements for determining the optimal operation boundaries for maximum $S_{I}$ as well as the best $B_{\text {min. }}$. 


\section{Dynamic range measurement of GHE}

(a)
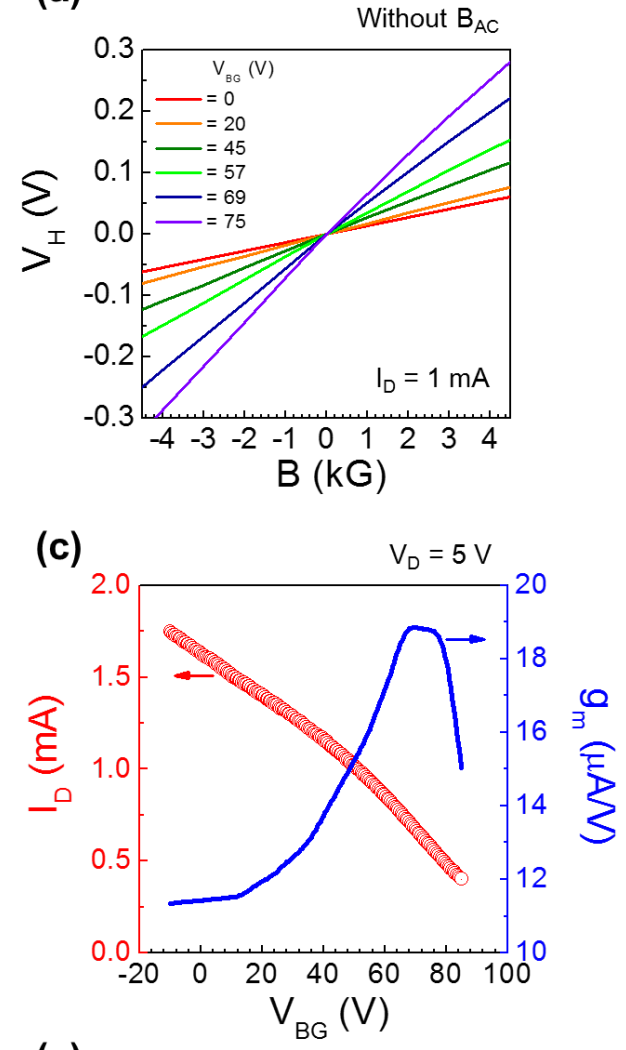

(e)

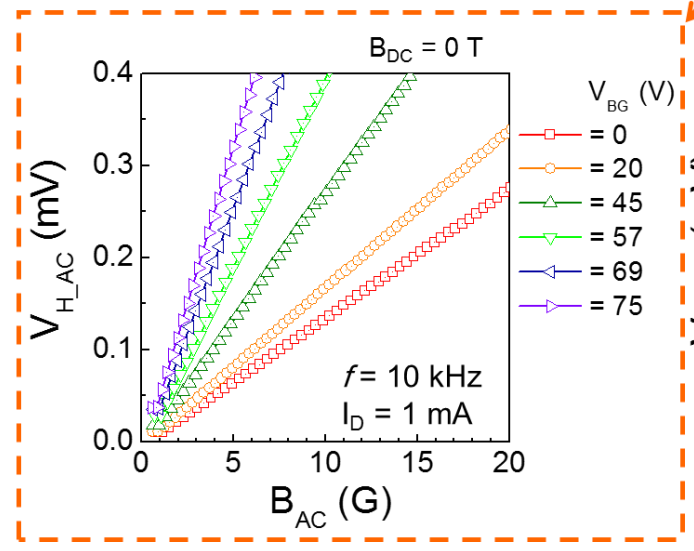

(b)

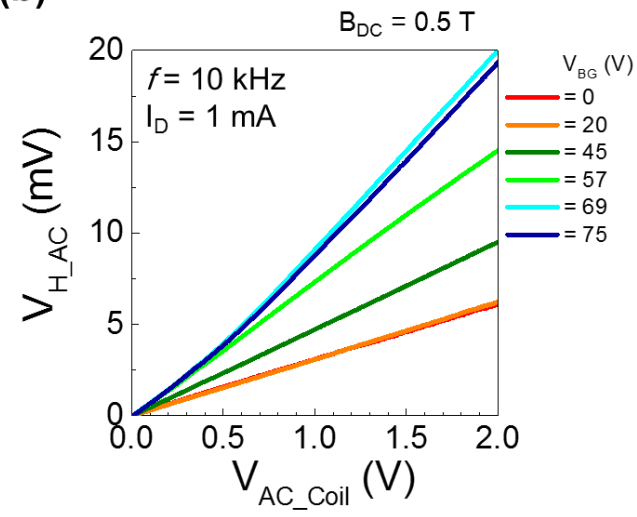

(d)

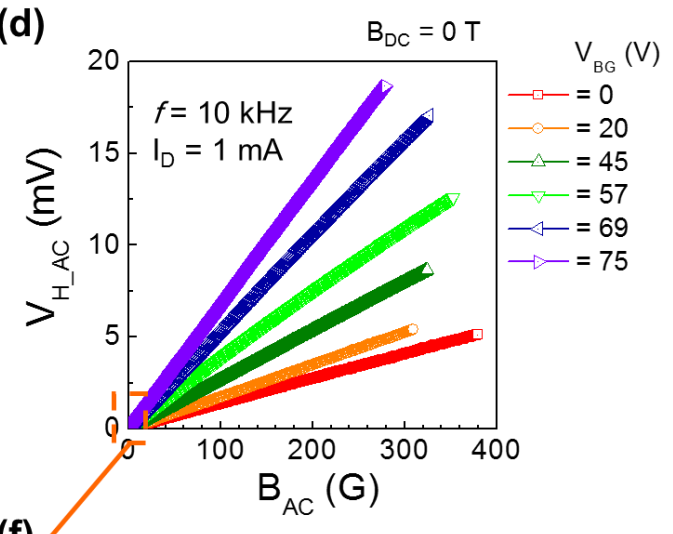

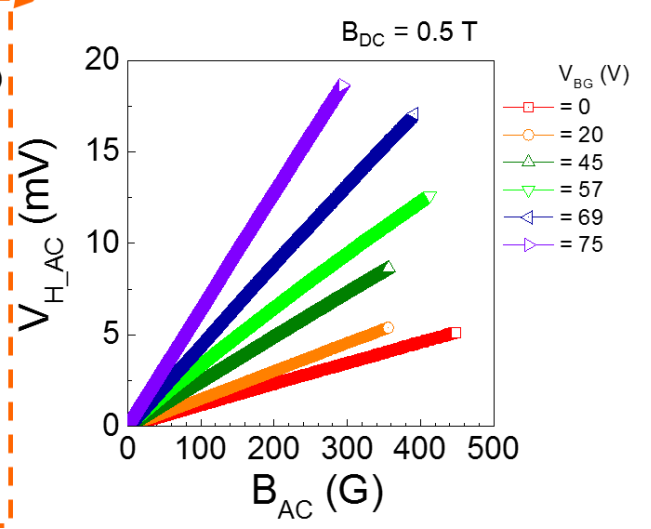

Figure S6. Dynamic range measurement of GHE. (a) The static $B$-field $\left(B_{D C}\right)$ dependence of $V_{H}$. (b) $V_{H_{-} A C}$ curves as a function of $V_{A C_{-} C o i l}$ for the calibration of $\mathrm{AC}$ magnetic field $\left(B_{A C}\right)$ generated by the coil. (c) $I_{D}-V_{B G}$ transfer curve (red symbols) at $V_{D}=5 \mathrm{~V}$ and the corresponding transconductance $g_{m}$ curve (blue line) for the tested GHE. (d) $B_{A C}$ dependence of $V_{H \_A C}$ at 
different $V_{B G}$ without magnetic field (i.e. $B_{D C}=0 \mathrm{~T}$ ). (e) Magnification of the graph in (d) for the small $B_{A C}$ ranges below $20 \mathrm{G}$. (f) $B_{A C}$ dependence of $V_{H_{-} A C}$ at different $V_{B G}$ with $B_{D C}=0.5 \mathrm{~T}$.

For the dynamic range measurement of GHE, the fundamental magnetic and electrical properties of the measurement setup and the sample are characterized. Figure S6a displays the static $B$-field $\left(B_{D C}\right)$ dependence of $V_{H}$ of the tested GHE sample. Each slope indicates the Hall effect sensitivity with respect to a different $V_{B G}$ at $I_{D}=1 \mathrm{~mA}$. Figure S6b displays the $B_{A C}$ dependent $V_{H_{-} A C}$ as a function of $V_{B G}$ at $B=0.5 \mathrm{~T}(f=10 \mathrm{kHz})$. Using these two figures, the small AC magnetic field $\left(B_{A C}\right)$ generated by the copper wire coil located on top of the sample (which is illustrated in Figure 7(a) in the main text) is calibrated, giving the relation between $V_{A C_{-} \text {Coil }}$ and $B_{A C}$. 


\section{Demonstration of the GHE magnetic sensor operation with a commercial microcontroller board}

GHE magnetic sensor is directly connected to the $5 \mathrm{~V}$ DC source of Arduino ${ }^{4}$, the commercial programmable microcontroller board, and the Hall voltage $\left(\mathrm{V}_{\mathrm{H}}=\mathrm{V}+-\mathrm{V}^{-}\right)$variation is detected from the analogue pin of Arduino without any signal amplifier. When three neodymium magnet is loaded on the end of a household hand-drill and rotated on top of the GHE sensor, their magnetic field variation induces the change of $\mathrm{V}_{\mathrm{H}}$ within ranging of $10-15 \mathrm{mV}$. This difference is enough to be recognized as an on-/off-state of GHE device compared with ambient noise level of $\sim 4 \mathrm{mV}$ (See the Supplementary Video for the demonstration).

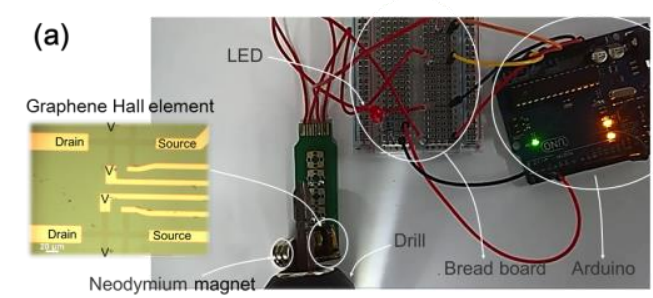

(b)

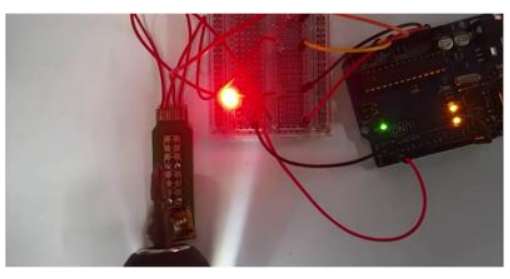

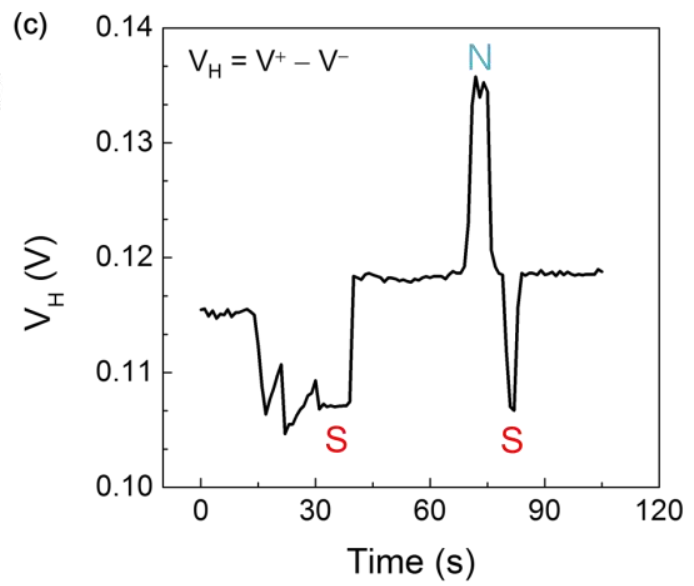

Figure S7. Feasibility of GHE as a magnetic sensor application. (a) Experimental schematic for GHE as a practical magnetic sensor using open source hardware of Arduino. Commercial neodymium magnets are loaded onto an electrical motor drill. In case of the neodymium magnets are perpendicular to GHE, a red commercial light emitting diode is then turned on as displayed in Figure S7(b). The inset displays an optical microscope image of fabricated GHE. (c) The transient data obtained from the Arduino. 


\section{REFERENCES}

1 Schroder, D. K. Semiconductor Material and Device Characterization; John Wiley \& Sons, 2006.

2 Von Haartman, M. \& Östling, M. Low-Frequency Noise in Advanced MOS Devices; Springer Science \& Business Media, 2007.

3 Balandin, A. A. Low-Frequency 1/f Noise in Graphene Devices. Nat. Nanotechnol. 8, 549-555 (2013).

4 Barrett, S. Arduino Microcontroller Processing for Everyone; Morgan \& Claypool, 2010 